\title{
Note on the gauge symmetries of unimodular gravity
}

\author{
Enrique Álvarez, ${ }^{1,2, *}$ Sergio González-Martín, ${ }^{1,2, \dagger}$ and Carmelo P. Martín ${ }^{3, *}$ \\ ${ }^{1}$ Instituto de Física Teórica, IFT-UAM/CSIC, Universidad Autónoma, 28049 Madrid, Spain \\ ${ }^{2}$ Departamento de Física Teórica, Universidad Autónoma de Madrid, 28049 Madrid, Spain \\ ${ }^{3}$ Universidad Complutense de Madrid (UCM), Departamento de Física Teórica I, \\ Facultad de Ciencias Físicas, Av. Complutense S/N (Ciudad Univ.), 28040 Madrid, Spain \\ (Received 26 April 2016; published 30 June 2016)
}

The symmetries of unimodular gravity are clarified somewhat.

DOI: 10.1103/PhysRevD.93.123018

\section{INTRODUCTION}

Unimodular gravity (UG) is a truncation of general relativity (GR) in the sense that only unimodular metrics (i.e. those with unit determinant) are considered. A recent review is [1], where some early references can be found. It is remarkable that Einstein himself proposed a closely related theory in 1919 [2].

The theory can be (and it is technically convenient) formulated in such a way that it has an added Weyl invariance by writing

$$
\hat{g}_{\mu \nu} \equiv\left(T_{U} g\right)_{\mu \nu} \equiv|g|^{-\frac{1}{n}} g_{\mu \nu}
$$

(where $g \equiv \operatorname{det} g_{\alpha \beta}$ ). The reason is that then the variations $\delta g_{\alpha \beta}$ are unconstrained, whereas the variations of the unimodular metric have got to be traceless:

$$
\hat{g}^{\alpha \beta} \delta \hat{g}_{\alpha \beta}=0 .
$$

We shall denote the mapping,

$$
\mathrm{UR}: g_{\mu \nu} \rightarrow \hat{g}_{\alpha \beta},
$$

as "unimodular reduction." It is not invertible, since there is no way to reconstruct $g_{\alpha \beta}$ from its unimodular reduction $\hat{g}_{\alpha \beta}$. On the other hand, once we restrict the theory to unimodular metrics, the ensuing theory (UG) is not invariant under the full diffeomorphism group of the manifold $\operatorname{Diff}(M)$, but only under the subgroup that preserves the unimodularity condition, which we have dubbed $\operatorname{TDiff}(M)$. This is essentially what mathematicians call the volume preserving subgroup [3]. It has been pointed out that this symmetry is enough to kill the three unwanted polarizations when defining the massless theory from a massive theory in flat space [4]. At any rate, under unimodular reduction EinsteinHilbert action gets transformed into

\footnotetext{
*enrique.alvarez@uam.es sergio.gonzalez.martin@csic.es *carmelop@fis.ucm.es
}

$$
\begin{aligned}
\mathrm{UR}: S_{\mathrm{GR}} & \equiv-M_{P}^{n-2} \int d^{n} x \sqrt{|g|} R\left[g_{\alpha \beta}\right] \rightarrow \\
\rightarrow S_{\mathrm{UG}} & \equiv-M_{P}^{n-2} \int d^{n} x R\left[\hat{g}_{\alpha \beta}\right],
\end{aligned}
$$

and the unimodular action in terms of unconstrained variables reads

$$
S_{\mathrm{UG}}=-M_{P}^{n-2} \int d^{n} x|g|^{\frac{1}{n}}\left(R+\frac{(n-1)(n-2)}{4 n^{2}} \frac{\partial_{\mu} g \partial^{\mu} g}{g^{2}}\right) .
$$

Once here, one can never go back to the Einstein frame as this action is Weyl invariant.

In terms of this unconstrained metric, the equations of motion (EM) are given by the manifestly traceless expression [5]

$$
\begin{aligned}
R_{\mu \nu}-\frac{1}{n} R g_{\mu \nu}= & \Theta_{\mu \nu} \\
\Theta_{\mu \nu} \equiv & \frac{(n-2)(2 n-1)}{4 n^{2}}\left(\frac{\partial_{\mu} g \partial_{\nu} g}{g^{2}}-\frac{1}{n} \frac{(\partial g)^{2}}{g^{2}} g_{\mu \nu}\right) \\
& -\frac{n-2}{2 n}\left(\frac{\partial_{\mu} \partial_{\nu} g-\Gamma_{\mu \nu}^{\lambda} \partial_{\lambda} g}{g}\right. \\
& \left.-\frac{1}{n} g^{\alpha \beta} \frac{\partial_{\alpha} \partial_{\beta} g-\Gamma_{\alpha \beta}^{\lambda} \partial_{\lambda} g}{g} g_{\mu \nu}\right) .
\end{aligned}
$$

The explicit presence of the determinant of the metric, $g$, clearly indicates the EM are not Diff invariant.

Now given the fact that the EM are Weyl invariant, we can always transform from $g_{\mu \nu}$ to $\bar{g}_{\mu \nu}$ such that

$$
\bar{g}=1,
$$

where the EM simply read

$$
\bar{R}_{\mu \nu}=\frac{1}{n} \bar{R} \bar{g}_{\mu \nu} .
$$

The solutions of these equations are, by definition, Einstein spaces [6]. The Bianchi identities in the absence of torsion do imply then $\partial_{\mu} R=0$.

Given a unimodular Einstein space, $\bar{g}_{\mu \nu}$, all its Weyl rescalings, 


$$
g_{\mu \nu} \equiv \Omega^{2}(x) \bar{g}_{\mu \nu},
$$

are also solutions of the equations [(6)]. They span a Weyl orbit of solutions. In four dimensions, it is well known that the necessary and sufficient condition [7] for a space to be conformally Einstein is for it to be Bach flat,

$$
B_{\mu \nu} \equiv \nabla^{\alpha} \nabla^{\beta} W_{\alpha \mu \nu \beta}-\frac{1}{2} R^{\alpha \beta} W_{\alpha \mu \nu \beta}=0,
$$

where $W_{\mu \nu \rho \sigma}$ is the Weyl tensor. We are not aware of a similar statement in arbitrary dimension.

The full symmetry group of this action is quite large though, incorporating Weyl transformations of the metric. This means that, in the process of the unimodular reduction of the Einstein-Hilbert action, the symmetry group changes, namely,

$$
\text { UR: } \operatorname{Diff}(M) \rightarrow \operatorname{TDiff}(M) \ltimes \operatorname{Weyl}(M) .
$$

Let us examine this process of symmetry reduction in more detail. We shall be cavalier about domains of definition of the transformations, and all of our reasoning will be purely local.

\section{TDIFF INVARIANCE OF THE UNIMODULAR ACTION}

It is not immediately obvious in which reference systems the EM [3] are valid.

Let us first start with the analysis of the already mentioned change of the symmetry group in the process of unimodular reduction.

We can represent a linearized element of $\operatorname{Diff}_{0}(M)$ (the subgroup of $\operatorname{Diff}(M)$ connected with the identity) as

$$
x \rightarrow x^{\prime} \equiv x+\xi
$$

The corresponding Jacobian matrix is

$$
J_{\beta^{\prime}}^{\alpha}(x) \equiv \frac{\partial x^{\alpha}}{\partial x^{\beta^{\prime}}},
$$

and its determinant will be denoted by the letter $J$.

The determinant of the metric then transforms as

$$
g(x) \rightarrow g^{\xi}(x+\xi)=J^{2}(x) g(x) .
$$

And for the case of a volume-preserving diffeomorphism, it is transverse in the sense that

$$
\partial_{\lambda} \xi_{T}^{\lambda}=0
$$

and the Jacobian matrix is itself unimodular,

$$
J_{T}=1 .
$$

Let us examine what happens with the action of $\operatorname{TDiff}_{0} \ltimes \operatorname{Weyl}(M)$. Clearly,

$$
g_{\mu \nu}^{\xi}(x) \equiv J_{\mu}^{\alpha} J_{\nu}^{\beta} g_{\alpha \beta}(x-\xi),
$$

and, consequently,

$$
g_{\mu \nu}^{\xi \Omega} \equiv \Omega^{2}(x) J_{\mu}^{\alpha} J_{\nu}^{\beta} g_{\alpha \beta}(x-\xi) .
$$

On the other hand, the other way around,

$$
g_{\mu \nu}^{\Omega \xi}(x)=\Omega^{2}(x-\xi) J_{\mu}^{\alpha} J_{\nu}^{\beta} g_{\alpha \beta}(x-\xi) .
$$

This corresponds to the noncommutativity of the diagram,

$$
\begin{aligned}
& g_{\mu \nu} \stackrel{\text { TDiff }}{\longrightarrow} g_{\mu \nu}^{\xi} \\
& \downarrow_{\text {Weyl }} \downarrow \text { Weyl } \\
& g_{\mu \nu}^{\Omega} \stackrel{\text { TDiff }}{\longrightarrow} g_{\mu \nu}^{\xi \Omega} \neq g_{\mu \nu}^{\Omega \xi}
\end{aligned}
$$

which is why the symmetry group is a semidirect product.

We can move now to answer the question of the validity of the EM of UG.

The two possible paths when going from GR to UG are shown in the following diagram:

$$
\begin{array}{cc}
G R \stackrel{\text { Diff }}{\longrightarrow} G R \\
\downarrow_{U R} \quad \downarrow_{U R} \\
U G \stackrel{\text { Diff }}{\longrightarrow} U G
\end{array}
$$

The rightmost path corresponds to, first perform a Diff

$$
g_{\mu \nu}^{\xi}(x) \equiv\left(T_{\xi} g\right)_{\mu \nu}(x) \equiv J_{\mu}^{\alpha}(x-\xi) J_{\nu}^{\nu}(x-\xi) g_{\alpha \beta}(x-\xi)
$$

and unimodularly reduce afterwards. The corresponding unimodular metric is then

$$
\left(T_{U} T_{\xi} g\right)_{\mu \nu}(x)=J^{-\frac{2}{n}}(x) g^{-\frac{1}{n}}(x)\left(T_{\xi} g\right)_{\mu \nu} .
$$

Let us now perform an arbitrary diffeomorphism after unimodular reduction (corresponding to the left path in the diagram). The result is

$$
\left(T_{\xi} T_{U} g\right)_{\alpha \beta}(x)=J^{-\frac{2}{n}}(x-\xi) g^{-\frac{1}{n}}(x-\xi)\left(T_{\xi} g\right)_{\alpha \beta} .
$$

This means again that the diagram above is not commutative.

Indeed, we find it particularly clarifying to examine what happens in this latter case.

If we perform a Diff in (6), the determinant $g(x)$ transforms as 


$$
\begin{aligned}
\partial_{\lambda^{\prime}} g^{\xi}\left(x^{\prime}\right) & =J_{\lambda^{\prime}}^{\alpha} \partial_{\alpha}\left(J^{2}(x) g(x)\right) \\
& =J_{\lambda^{\prime}}^{\alpha}\left(J^{2} \partial_{\alpha} g(x)+2 g(x) J(x) \partial_{\alpha} J\right),
\end{aligned}
$$

which conveys the fact that the first monomial in the EM transforms as

$$
\begin{aligned}
\frac{\partial_{\mu^{\prime}} g^{\xi}\left(x^{\prime}\right) \partial_{\nu^{\prime}} g^{\xi}\left(x^{\prime}\right)}{g^{\prime}\left(x^{\prime}\right)^{2}} & =\frac{J_{\mu^{\prime}}^{\alpha} J_{\nu^{\prime}}^{\beta}}{J^{4} g^{2}}\left(\partial_{\alpha} g J^{2}+2 g J \partial_{\alpha} J\right)\left(J^{2} \partial_{\beta} g+2 g J \partial_{\beta} J\right) \\
& =J_{\mu^{\prime}}^{\alpha} J_{\nu^{\prime}}^{\beta}\left\{\frac{\partial_{\alpha} g \partial_{\beta} g}{g^{2}}+2 \frac{\partial_{\alpha} J \partial_{\beta} g+\partial_{\alpha} g \partial_{\beta} J}{J g}+4 \frac{\partial_{\alpha} J \partial_{\beta} J}{J^{2}}\right\},
\end{aligned}
$$

and its trace, which is the one subtracted from it in (6), is just

$$
\left(\frac{\partial_{\alpha} g}{g}+2 \frac{\partial_{\alpha} J}{J}\right)^{2}
$$

The second monomial transforms, in turn, as

$$
\begin{aligned}
\frac{\partial_{\mu^{\prime}} \partial_{\nu^{\prime}} g^{\xi}(x+\xi)}{g^{\xi}(x+\xi)} & =\frac{J_{\mu^{\prime}}^{\rho} J_{\nu^{\prime}}^{\alpha} \partial_{\rho}}{J^{2} g}\left(J^{2} \partial_{\alpha} g(x)+2 g(x) J(x) \partial_{\alpha} J\right) \\
& =J_{\mu^{\prime}}^{\rho} J_{\nu^{\prime}}^{\alpha}\left\{2 \frac{\partial_{\rho} J}{J} \frac{\partial_{\alpha} g}{g}+\frac{\partial_{\rho} \partial_{\alpha} g}{g}+2 \frac{\partial_{\rho} g}{g} \frac{\partial_{\alpha} J}{J}+2 \frac{\partial_{\alpha} J}{J} \frac{\partial_{\rho} J}{J}+2 \frac{\partial_{\rho} \partial_{\alpha} J}{J}\right\},
\end{aligned}
$$

with its trace now

$$
4 \frac{\partial_{\alpha} J \partial^{\alpha} g}{g J}+\frac{\partial^{2} g}{g}+2 \frac{\partial_{\alpha} J \partial^{\alpha} J}{J^{2}}+2 \frac{\partial^{2} J}{J}
$$

\section{CONCLUSIONS}

When performing a general $\operatorname{Diff}_{0}(M)$ transformation in the unimodular EM, the extra terms generated are

$$
\begin{aligned}
\operatorname{EM}\left[g_{\mu \nu}^{\xi}\right]_{\alpha^{\prime} \beta^{\prime}} & =J_{\alpha^{\prime}}^{\alpha} J_{\beta^{\prime}}^{\beta}\left\{\operatorname{EM}\left[g_{\mu \nu}\right]_{\alpha \beta}+\frac{n-2}{2 n}\left(\frac{1}{n} \frac{\partial_{\alpha} J \partial_{\beta} g+\partial_{\alpha} g \partial_{\beta} J}{J g}\right.\right. \\
& \left.+2 \frac{1-n}{n} \frac{\partial_{\alpha} J \partial_{\beta} J}{J^{2}}+2 \frac{\partial_{\alpha} \partial_{\beta} J}{J}\right) \\
& \left.-\frac{n-2}{n^{2}}\left(\frac{1}{n} \frac{\partial_{\mu} J \partial^{\mu} g}{g J}+\frac{1-n}{n} \frac{\partial_{\mu} J \partial^{\mu} J}{J^{2}}+\frac{\partial^{2} J}{J}\right) g_{\alpha \beta}\right\} .
\end{aligned}
$$

To be specific, the fact that a given metric $g_{\mu \nu}$ is a solution of the unimodular equations of motion does not imply that it remains a solution after an arbitrary diffeomorphism $\xi \in \operatorname{Diff}_{0}(M)$ unless, of course, this happens to be transverse, $\xi \in \operatorname{TDiff}_{0}(M)$. Certainly, there is no problem with performing the Diff before the unimodular reduction, since GR is invariant as shown in the last diagram.

In other words, the assertion that a given metric is a solution of the UG equations of motion is not Diff(M) invariant, but only $\operatorname{TDiff}(\mathrm{M}) \ltimes \operatorname{Weyl}(\mathrm{M})$ invariant. Is there a coordinate system which is not attainable through a symmetry transformation? By the following argument, one might think that there is none. An arbitrary diffeomorphism acts as

$$
g_{\mu \nu}^{\xi}(x) \equiv J_{\mu}^{\alpha} J_{\nu}^{\beta} g_{\alpha \beta}(x-\xi),
$$

which has the same number of parameters as the action of a volume-preserving diffeomorphism composed with a Weyl transformation. However, there is a subtlety here, since one should have solutions to the equation,

$$
\frac{J_{\mu}^{\alpha}}{J}=\frac{\partial y^{\alpha}}{\partial x^{\mu}},
$$

and this is possible only when

$$
\partial_{\nu}\left(\frac{J_{\mu}^{\alpha}}{J}\right)=\partial_{\mu}\left(\frac{J_{\nu}^{\alpha}}{J}\right)
$$

which will not, in general, be true.

In the Appendix, we work out a simple example to illustrate this fact.

\section{ACKNOWLEDGMENTS}

The origin of this work lies in a question by Gerardus 't Hooft at the Shapes of Gravity meeting held in Nijmegen in 2016. It has been partially supported by the European Union FP7 ITN INVISIBLES (Marie Curie Actions, PITNGA-2011- 289442) and (HPRN-CT-200-00148); COST action MP1405 (Quantum Structure of Spacetime), COST action MP1210 (The String Theory Universe) as well as by 
FPA2012-31880 (MICINN, Spain), FPA2014-54154-P (MICINN, Spain), and S2009ESP-1473 (CA Madrid). This project has received funding from the European Union's Horizon 2020 research and innovation program under the Marie Sklodowska-Curie Grant Agreement No. 690575. This project has also received funding from the European Union's Horizon 2020 research and innovation program under the Marie Sklodowska-Curie Grant Agreement No. 674896. The authors acknowledge the support of the Spanish MINECO Centro de Excelencia Severo Ochoa program under Grant No. SEV-2012-0249.

\section{APPENDIX A: ANY METRIC CAN BE MADE UNIMODULAR BY A DIFFEOMORHISM}

All we have to do is to find a solution of the equation

$$
J(x)=\frac{1}{g(x)^{2}} .
$$

At the linear level (algebra),

$$
\partial_{\mu} \xi^{\mu}=\frac{1}{g(x)^{2}}-1
$$

and this is trivially solved in a formal way by

$$
\xi^{\mu}(x)=\partial^{\mu} \square^{-1}\left(\frac{1}{g(x)^{2}}-1\right),
$$

whose solution is unique under essentially the same conditions as the corresponding solution of the wave equation [8].

\section{APPENDIX B: FLAT SPACE IN CYLINDRICAL COORDINATES IS NOT A SOLUTION OF UG}

Let us consider, to be specific, the ordinary threedimensional Euclidean space $\mathbb{R}^{3}$ in cylindrical coordinates:

$$
d s^{2}=d r^{2}+r^{2} d \theta^{2}+d z^{2} .
$$

A simple calculation, taking into account that

$$
\begin{gathered}
\Gamma_{\phi \phi}^{r}=-r \\
\Gamma_{\phi r}^{\phi}=\frac{1}{r},
\end{gathered}
$$

yields

$$
\Theta_{\mu \nu}=\frac{1}{27 r^{2}}\left(\begin{array}{ccc}
-7 & 0 & 0 \\
0 & 8 r^{2} & 0 \\
0 & 0 & -1
\end{array}\right) \neq 0 .
$$

That is, flat space in cylindrical coordinates is not a solution of the unimodular equations of motion. This happens, of course, because the transformation from Cartesian to cylindrical coordinates does not belong to $\operatorname{TDiff}\left(\mathbb{R}^{3}\right)$. In terms of the integrability conditions (33), it is clear that there is no integrating factor because the Jacobian from Cartesian coordinates reads

$$
J_{\mu}^{\alpha} \equiv\left(\begin{array}{ccc}
\cos \theta & \sin \theta & 0 \\
-r \sin \theta & r \cos \theta & 0 \\
0 & 0 & 1
\end{array}\right),
$$

its determinant is

$$
J=r,
$$

and the integrability conditions fail here because, for example,

$$
\frac{\partial}{\partial \theta}\left(\frac{J_{r}^{x}}{J}\right)=-\frac{\cos \theta}{r^{2}} \neq \frac{\partial}{\partial r}\left(\frac{J_{\theta}^{x}}{J}\right)=0 .
$$

[1] E. Álvarez, S. González-Martín, M. Herrero-Valea, and C. P. Martín, Unimodular gravity redux, Phys. Rev. D 92, 061502 (2015).

[2] A. Einstein, Do gravitational fields play an essential part in the structure of the elementary particles of matter?, Sitzungsber. Preuss. Akad. Wiss. Phys. Math. K1. 1919, 433 (1919).

[3] D. McDuff, On the group of volume-preserving diffeomorphisms of $\mathbb{R}^{n}$, Trans. Am. Math. Soc. 261, 103 (1980).

[4] J. J. van der Bij, H. van Dam, and Y. J. Ng, The exchange of massless spin two particles, Physica A (Amsterdam) 116A, 307 (1982).
[5] E. Álvarez, S. González-Martín, M. Herrero-Valea, and C. P. Martín, Quantum corrections to unimodular gravity, J. High Energy Phys. 08 (2015) 078.

[6] A. L. Besse, Einstein Manifolds (Springer, New York, 2007).

[7] C. N. Kozameh, E. T. Newman, and K. P. Tod, Conformal Einstein spaces, Gen. Relativ. Gravit. 17, 343 (1985).

[8] F. G. Friedlander, The Wave Equation on a Curved Space-Time (Cambridge, Cambridge University Press, 2010). 